\title{
Micromicetos del suelo de una plantación de plátano (Musa paradisiaca) en Teapa, Tabasco, México
}

\author{
Soil microfungi from a banana (Musa paradisiaca) plantation in Teapa, Tabasco, Mexico
}

\author{
Mariana Del Olmo-Ruiz ${ }^{1,2 *}$, Joaquín Cifuentes-Blanco ${ }^{1}$, Guadalupe Vidal-Gaona ${ }^{1}$ y Edmundo Rosique-Gil ${ }^{3}$ \\ ${ }^{1}$ Herbario FCME, Sección de Micología, Facultad de Ciencias, Universidad Nacional Autónoma de México, Apartado postal 70-399, Ciudad \\ Universitaria, 04510 México, D.F., México. \\ ${ }^{2}$ Dirección actual: Division of Plant Pathology and Microbiology, Department of Plant Sciences, University of Arizona, 1140 E. South Campus Drive, \\ Forbes 303, Tucson, AZ, 85721, USA. \\ ${ }^{3}$ Departamento de Botánica, Instituto de Biología, Universidad Nacional Autónoma de México, Apartado postal 70-233, Ciudad Universitaria, 04510 \\ México, D.F., México. \\ *Correspondencia: mdelolmo@email.arizona.edu
}

\begin{abstract}
Resumen. Se analizó la micobiota presente en el suelo de un cultivo de plátano (Musa paradisiaca L.) en el municipio de Teapa en el estado de Tabasco, México. Los objetivos fueron conocer las especies habitantes de este suelo y la dinámica de la comunidad con respecto a la composición de especies a lo largo de un año. Se llevaron a cabo 4 muestreos durante temporadas distintas y los aislamientos se realizaron mediante la técnica de dilución en placa. Se recuperaron 91 colonias, correspondientes a 30 especies diferentes de hongos mitospóricos, de las cuales únicamente Aspergillus flavus Link, Fusarium nivale (Fr.) Ces y Trichoderma harzianum Rifai fueron especies residentes de ese suelo y el resto, esporádicas. El índice de similitud de Sorensen evidenció una sucesión de especies entre las temporadas de muestreo. Del total de especies determinadas en este estudio, 8 no habían sido encontradas como habitantes de suelos mexicanos. Asimismo, se mostró que el suelo analizado es un hábitat muy rico en micromicetos y que es necesario incrementar los esfuerzos de colecta para tener un mayor conocimiento sobre la diversidad en nuestro país.
\end{abstract}

Palabras clave: riqueza, hongos, mitospóricos, sucesión.

\begin{abstract}
In this study, we analyzed the soil microfungal community from a banana (Musa paradisiaca L.) plantation in Teapa, a municipality of Tabasco State in Mexico. The objectives were to determine the fungal species present and to analyze the community composition throughout an entire year. We performed 4 samplings during different seasons and the fungal isolates were recovered using the dilution plate technique. We isolated 91 strains from 30 different mitosporic species where only Aspergillus flavus Link, Fusarium nivale (Fr.) Ces and Trichoderma harzianum Rifai were considered as resident species while the remaining species were sporadic. The Sorensen similarity index suggested that species succession had occurred among the sampling seasons. From all the species identified in this survey, 8 had not been found as inhabitants of Mexican soils. The study shows the high microfungal richess of this soil and highlights the importance of Mexican soils as sources of mitosporic fungal species.
\end{abstract}

Key words: richness, fungi, mitosporic, succession.

\section{Introducción}

En México se han realizado diversas investigaciones sobre micromicetos de suelos recolectados en distintas regiones del país, entre las que figuran varias localidades a lo largo de la Carretera Panamericana (Wolf, 1939), distintos sitios de zonas tropicales y subtropicales del país (Zambrano y Casas- Campillo, 1959), el desierto de Sonora (Ranzoni, 1968), localidades de Nuevo León, Tamaulipas, Michoacán e Hidalgo (Shanor, 1942; Céspedes y Castillo,

Recibido: 05 marzo 2008; aceptado: 04 septiembre 2009
1982), la pared occidental del volcán Popocatépetl (Rodríguez, 1984) y 3 localidades de La Joya del Obispo en Oaxaca (Piñón , 1984). Además se ha caracterizado la micobiota presente en suelos que sostienen diferentes tipos de vegetación, como bosque mesófilo de montaña (Heredia, 1999), bosque de coníferas (Heredia et al., 2001; Bills et al., 2001), un tintal (Rosique, 2004) y algunos suelos cultivados con sorgo (Reyes y Castillo, 1981), nogal (Samaniego et al., 1988) y trigo (Velásquez, 1989).

En cada uno de estos estudios se registran diferentes valores en la riqueza de micromicetos que oscilan entre 1 (Shanor, 1942) y 65 (Rodríguez, 1984), y a pesar de que 
se encontraron constantemente algunas especies en los distintos tipos de suelos, ninguno de los listados presenta la misma composición, lo que indica que distintos suelos contienen diferentes comunidades de hongos microscópicos y que aún estamos lejos de conocer las especies fúngicas habitantes de los suelos mexicanos.

Los objetivos del presente trabajo fueron determinar las especies presentes en un suelo cultivado con plátano (Musa paradisiaca L.) en el municipio de Teapa, Tabasco, a lo largo de un año de colecta y comparar la composición de las especies fúngicas encontradas durante ese tiempo.

\section{Materiales y métodos}

El trabajo de campo se realizó en los campos de cultivo del Centro de Investigación de Plátano y Cultivos Tropicales, dependencia del Gobierno del estado de Tabasco, ubicado en el municipio de Teapa (Fig. 1).

Teapa se encuentra localizado a $17^{\circ} 32^{\prime} \mathrm{N}, 92^{\circ} 57^{\prime} \mathrm{O}$, a $40 \mathrm{~m}$ snm, cuenta con un clima cálido húmedo con lluvias todo el año, y sus principales tipos de vegetación son los pastizales, las asociaciones tular-popal, los campos agrícolas, la selva y los manglares (INEGI, 2001). En el caso de los campos agrícolas, el plátano se considera uno de los cultivos más importantes y junto con la caña de azúcar y el cacao constituye una pieza clave en la economía de la región (CEA, 1999).

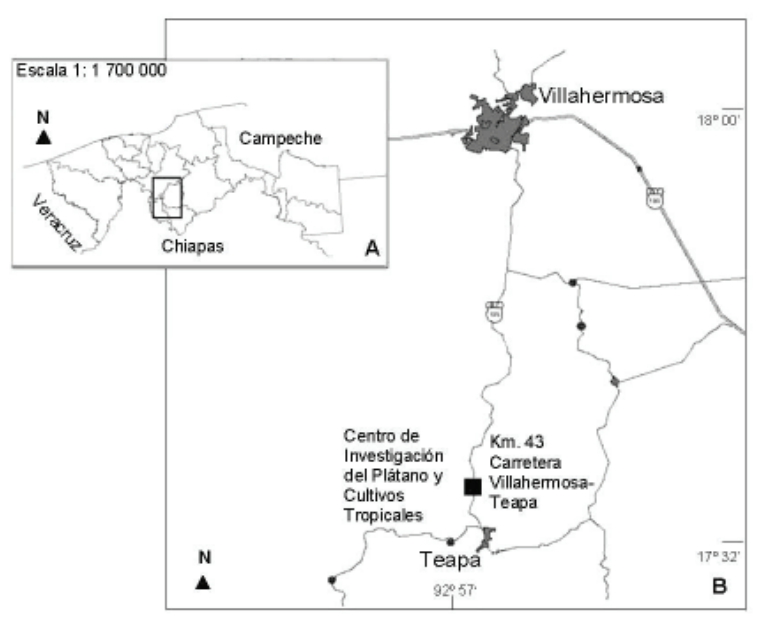

Figura 1. Ubicación geográfica. del estado de Tabasco (A) y del Centro de Investigación del Plátano y Cultivos Tropicales (B), Km. 43 de la carretera Villahermosa- Teapa, municipio de Teapa, Tabasco, México.
Se realizaron 4 muestreos durante las siguientes fechas: agosto y noviembre de 2001 y febrero y mayo de 2002. Las colectas consistieron en tomar del mismo sitio $200 \mathrm{~g}$ del horizonte A del suelo con ayuda de una espátula previamente esterilizada a la flama con etanol al 96\%. Las muestras fueron depositadas en bolsas de plástico estériles, conservándolas a una temperatura de $5^{\circ} \mathrm{C}$ hasta su procesamiento posterior en el laboratorio (24 horas aproximadamente) (Alef y Nannipieri, 1995; Davet y Rouxel, 2000). Una vez en la laboratorio, utilizando el método de dilución en placa (Davet y Rouxel, 2000) e iniciando con un gramo de suelo seco, se realizaron 3 diluciones en agua destilada estéril: 1:100; 1:1000 y 1:10000. Se tomó $1 \mathrm{ml}$ de la suspensión acuosa a partir de cada tubo de ensayo y se sembró el inóculo por triplicado en cajas de Petri con medios de cultivo de papa dextrosa agar (PDA) y agua agar (AA), adicionados con estreptomicina $(0.030 \mathrm{~g} / \mathrm{L})$ y rosa de bengala $(0.050 \mathrm{~g} / \mathrm{L})$ (Farrow, 1954; Davet y Rouxel, 2000).

Las placas de agar fueron incubadas a $25^{\circ} \mathrm{C}$ durante 28 días y se examinaron diariamente con la finalidad de evaluar el crecimiento fúngico. El aislamiento y purificación de las colonias se realizó en los medios de cultivo extracto de malta agar (EMA) $2 \%$ y PDA.

Inicialmente se determinó el género de las colonias aisladas, siguiendo las claves de Domsch et al. (1993) y Barnett y Hunter (1998), y posteriormente la especie, utilizando las claves de Raper y Fennell (1965), Klitch y Pitt (1988) para Aspergillus; Ho et al. (1999) para Cladosporium; Booth (1977), Nelson et al. (1983) para Fusarium; Pitt (2000) para Penicillium y Bissett (1984; 1991; 1991a) para Trichoderma.

La frecuencia de constancia se calculó para conocer la permanencia de las especies a lo largo del tiempo de acuerdo con la fórmula utilizada por Heredia (1999):

Frec. constancia $(\%)=$ Núm. muestreos (misma especie)

Núm. total de muestreos

Además se calculó el índice de similitud de Sorensen (ISS) para comparar la composición de las comunidades fúngicas entre pares de muestreos con la fórmula que establece Mueller-Dombois (1981):

$$
\mathrm{ISS}=\frac{2 C}{-}+\frac{2 C}{A+B} * 100
$$

donde $C$ es el número de especies comunes a las 2 muestras; $A$ son todas las especies de la primera muestra, y $B$ todas las de la segunda. 


\section{Resultados}

En total se aislaron 91 colonias de micromicetos, de las cuales se determinó, por lo menos, el género de 82 y 9 fueron excluidas del estudio porque no formaron estructuras de reproducción.

El listado obtenido quedó integrado por 30 especies de hongos mitospóricos (Cuadro 1), que han sido previamente registradas como habitantes comunes del suelo de varias regiones del mundo (Domsch et al., 1993). Corresponden a 8 géneros distintos: Fusarium (9 especies aisladas, 30\% del total), Trichoderma (6 especies aisladas, 20\%), Aspergillus y Penicillium (5 especies aisladas cada género,16.7\%), Cladosporium (2 especies aisladas, 6.7\%), Acremonium, Geotrichum y Nigrospora (1 especie aislada cada género, $3.3 \%$ ).

Durante el primer muestreo en agosto de 2001 se recuperaron 22 colonias pertenecientes a 12 especies distintas; en el segundo muestreo (noviembre 2001) ,21 colonias, de 10 especies; en el tercero (febrero de 2002), 17 colonias de 11 especies, y en el cuarto (mayo 2002), 31 colonias de 14 especies.

Con respecto a la presencia de las especies en los distintos muestreos realizados, ninguna se encontró durante todo el periodo de colecta y únicamente Aspergillus flavus Link, Fusarium nivale (Fr.) Ces y Trichoderma harzianum

Cuadro 1. Especies de micromicetos presentes en un suelo cultivado con plátano en Teapa, Tabasco y sus valores de frecuencia de constancia

\begin{tabular}{|c|c|c|c|c|c|}
\hline Especies & $\begin{array}{l}2001 \\
\text { Agosto }\end{array}$ & Noviembre & $\begin{array}{l}2002 \\
\text { Febrero }\end{array}$ & Mayo & $\begin{array}{l}\text { Frecuencia de } \\
\text { constancia } \%\end{array}$ \\
\hline Aspergillus flavus & $0 *$ & 1 & 1 & 1 & 75 \\
\hline Fusarium nivale & 1 & 0 & 1 & 1 & 75 \\
\hline Trichoderma harzianum & 1 & 0 & 1 & 1 & 75 \\
\hline Fusarium equiseti & 1 & 1 & 0 & 0 & 50 \\
\hline Geotrichum candidum & 1 & 1 & 0 & 0 & 50 \\
\hline Fusarium sambucinum & 0 & 1 & 1 & 0 & 50 \\
\hline Acremonium murorum & 0 & 1 & 0 & 1 & 50 \\
\hline Aspergillus niger & 0 & 1 & 0 & 1 & 50 \\
\hline Fusarium dimerum & 0 & 1 & 0 & 1 & 50 \\
\hline Aspergillus japonicus & 1 & 0 & 0 & 1 & 50 \\
\hline Aspergillus niveus & 1 & 0 & 0 & 1 & 50 \\
\hline Cladosporium cladosporioides & 0 & 0 & 1 & 1 & 50 \\
\hline Fusarium chlamydosporum & 0 & 0 & 1 & 1 & 50 \\
\hline Penicillium citrinum & 1 & 0 & 1 & 0 & 50 \\
\hline Cladosporium sp. & 1 & 0 & 0 & 0 & 25 \\
\hline Fusarium culmorum & 1 & 0 & 0 & 0 & 25 \\
\hline Fusarium oxysporum & 1 & 0 & 0 & 0 & 25 \\
\hline Penicillium fellutanum & 1 & 0 & 0 & 0 & 25 \\
\hline Penicillium variabile & 1 & 0 & 0 & 0 & 25 \\
\hline Nigrospora oryzae & 0 & 1 & 0 & 0 & 25 \\
\hline Penicillium commune & 0 & 1 & 0 & 0 & 25 \\
\hline Trichoderma virens & 0 & 1 & 0 & 0 & 25 \\
\hline Aspergillus terreus & 0 & 0 & 1 & 0 & 25 \\
\hline Fusarium solani & 0 & 0 & 1 & 0 & 25 \\
\hline Trichoderma citrinoviride & 0 & 0 & 1 & 0 & 25 \\
\hline Trichoderma longibrachiatum & 0 & 0 & 1 & 0 & 25 \\
\hline Fusarium merismoides & 0 & 0 & 0 & 1 & 25 \\
\hline Penicillium purpurogenum & 0 & 0 & 0 & 1 & 25 \\
\hline Trichoderma aureoviride & 0 & 0 & 0 & 1 & 25 \\
\hline Trichoderma parceramosus & 0 & 0 & 0 & 1 & 25 \\
\hline
\end{tabular}

*Los números indican la presencia (1) o ausencia (0) de la especie en cada muestreo. 
Rifai mostraron una frecuencia de constancia del $75 \%$. Del resto de las especies, 11 se presentaron en el $50 \%$ de las muestras y 16 en el $25 \%$.

En el Cuadro 2 se muestran los resultados obtenidos del índice de similitud de Sorensen, en el que se puede observar que los muestreos de febrero y mayo de 2002 son los más parecidos en cuanto a composición de especies, con $40 \%$ de similitud, mientras que los muestreos realizados en agosto y noviembre de 2001 únicamente compartieron el $18 \%$ de las especies.

\section{Discusión}

Debido a que el método de dilución en placa sobreestima la abundancia de las especies que forman grandes cantidades de esporas (Davet y Rouxel, 2000), no se consideró apropiado incluir en este estudio los resultados del conteo de colonias por especie; sin embargo, se registra la frecuencia de constancia, la cual es independiente del método de aislamiento.

Las especies que se encontraron en 3 de los 4 muestreos realizados, (frecuencia de constancia 75\%) y que pueden considerarse residentes de este suelo (especies recurrentes adaptadas a persistir en el sustrato; Heredia, 1999), fueron $A$. flavus que en México ha sido aislada de suelos de huertas de nogal (Samaniego et al., 1988) y de un bosque mesófilo de montaña (Heredia, 1999); $F$. nivale, que anteriormente se registró en suelos del desierto de Sonora (Ranzoni, 1968) y T. harzianum, recuperada de suelos de Veracruz (Heredia, 1999) y Tabasco (Rosique, 2004).

Las especies que presentaron una frecuencia de constancia de $50 \%$ y que se consideran especies esporádicas (especies que aparecen con poca frecuencia; Heredia, 1999) son 9: Acremonium murorum (Corda) W. Gams, registrado en suelos derivados de cenizas volcánicas (Rodríguez, 1984); Aspergillus japonicus Saito, aislado del suelo de un tintal (Rosique, 2004); Aspergillus niger van Tieghem, una de las especies más comunes de suelos mexicanos, que se ha encontrado en localidades de Sonora (Ranzoni, 1968), Nuevo León (Reyes y Castillo, 1981), Estado de México (Rodríguez,
1984) Oaxaca (Piñón, 1984), Torreón (Samaniego et al., 1988), Veracruz (Heredia, 1999) y Tabasco (Rosique, 2004); Cladosporium cladosporioides (Fres.) de Vries se ha encontrado en suelos cultivados con sorgo (Reyes y Castillo, 1981), en huertas de nogal (Samaniego et al., 1988), en un bosque mesófilo de montaña (Heredia, 1999) y en un tintal (Rosique, 2004); Penicillium citrinum Thom, aislado de suelos de Veracruz (Heredia, 1999) y Tabasco (Rosique, 2004); Fusarium dimerum Penzig, se ha encontrado en Torreón (Samaniego et al., 1988); Fusarium equiseti (Corda) Sacc sensu Gordon, recuperado de suelos cultivados con trigo (Velásquez, 1989), y Geotrichum candidum Link ex Leman, que se encontró en muestras de suelo de distintas regiones del país, como Baja California (Ranzoni, 1968), Nuevo León (Reyes y Castillo, 1981), Oaxaca (Piñón, 1984) y Tabasco (Rosique, 2004).

Las especies con frecuencias de constancia de $25 \%$ fueron 10: Fusarium oxysporum Schlecht. emend. Snyd. et Hans. y Aspergillus terreus Thom que se han aislado de suelos de Torreón (Samaniego et al., 1988); Penicillium fellutanum Biourge y Trichoderma aureoviride Rifai, registrados como habitantes de suelos de Veracruz (Heredia, 1999) y Tabasco (Rosique, 2004); Penicillium variabile Sopp, aislado de suelo de huertas de nogal (Samaniego et al., 1988), de un bosque mesófilo de montaña (Heredia, 1999) y de un tintal (Rosique, 2004); Fusarium solani (Mart.) Appel et Wollenw. emend. Snyd. et Hans., aislada de suelos derivados de cenizas volcánicas (Rodríguez, 1984) y de un tintal (Rosique, 2004); Trichoderma citrinoviride Rifai, T. Longibrachiatum Rifai y $T$. parceramosus Bissett se han aislado del suelo de un tintal en Tabasco (Rosique, 2004); y Penicillium purpurogenum Stoll se encontró en muestras de suelo de Baja California (Ranzoni, 1968), Oaxaca (Piñón, 1984) Estado de México (Rodríguez, 1984), Torreón (Samaniego et al., 1988) y Veracruz (Heredia, 1999).

Entre las especies encontradas en esta investigación destacan aquellas que, a nuestro saber, no se han registrado como habitantes de suelos mexicanos; tal es el caso de Aspergillus niveus Blochwitz que es constante en suelos de desiertos, pastizales (Christensen, 1981) y cultivos de varias regiones del mundo (Joffe, 1963; Joshi y Chauhan, 1982); Fusarium chlamydosporum Wollenw.

Cuadro 2. Valores de los índices de similitud de Sorensen entre los 4 muestreos

\begin{tabular}{lllll}
\hline & Agosto 2001 & Noviembre 2001 & Febrero 2002 & Mayo 2002 \\
\hline Agosto 2001 & & & & \\
Noviembre 2001 & 18,18 & & \\
Febrero 2002 & 26,09 & 28,57 & 40 & \\
Mayo 2002 & 30,77 & 33,33 & 40 \\
\hline
\end{tabular}


et Reinking, que es frecuente en suelos de áreas tropicales y subtropicales (Nelson et al., 1983; Domsch et al., 1993); Fusarium sambucinum Fuckel y F. culmorum (W.G. Smith) Sacc que se encuentran en suelos de pastizales y cultivos (Domsch y Gams, 1972); Nigrospora oryzae (Berkeley et Broome) Petch cuya fase sexual (Khuskia oryzae H.J. Huds.) se ha aislado de suelos cultivados con trigo en la India (Joshi y Chauhan, 1982); Penicillium commune Thom y Fusarium merismoides Corda se han aislado de suelos cultivados (Joffe, 1963; Domsch y Gams, 1972); y Trichoderma virens (Miller, Giddens et Foster) von Arx que se ha aislado de varios suelos en la región este de la selva amazónica al norte de Brasil (Pfenning, 1997).

Es dato importante la aparición de $F$. oxysporum en este suelo, ya que esta especie se ha registrado como saprobia, pero también como parásita facultativa, y en su forma specialis cubense ataca las raíces de las plantas de plátano causando considerables pérdidas económicas (Domsch y Gams, 1972). Sin embargo, este estudio reveló que la especie se comporta como esporádica, con una frecuencia de constancia de $25 \%$ y hasta el momento no se tiene registro de plantas enfermas en la región. Por otra parte, el muestreo no fue exhaustivo y los resultados sólo hacen referencia a los puntos específicos de colecta, lo que impide extrapolar estos resultados a todo el cultivo, además de que la técnica de dilución en placa no hace distinción entre los hongos que crecen activamente en el suelo y los que se encuentran en estado de latencia a manera de esporas u otras estructuras de resistencia, por lo que tampoco se podría asegurar que esta especie se encuentre creciendo activamente.

El índice de similitud de Sorensen indica que las especies encontradas en este suelo, no fueron las mismas durante todo el año de colecta sino que existió una sucesión de un muestreo a otro, donde el cambio de especies más drástico ocurrió entre agosto de 2001 y noviembre del mismo año, ya que sólo se encontraron 2 especies compartidas, F. equiseti y $G$. candidum, que no volvieron a aparecer en ninguno de los demás muestreos. En el caso de la comparación entre el muestreo de noviembre de 2001 y febrero de 2002 el porcentaje de similitud aumentó hasta un $28 \%$.

Finalmente, los muestreos realizados en febrero y mayo de 2002 tuvieron el $40 \%$ de las especies en común. Estos resultados indican que la comunidad fúngica de este suelo sufrió cambios continuos en la composición de especies, tal vez como respuesta a la variación en los parámetros ambientales (Wicklow y Whittingham, 1974) o bien a las interacciones bióticas presentes; sin embargo, en este estudio es imposible determinar la causa debido a que no se tomaron datos de ninguno de esos factores. Por lo tanto, resulta importante realizar más muestreos a lo largo del año para poder determinar de mejor manera la sucesión de especies en este suelo.

En conclusión, esta investigación muestra que es posible recuperar un buen número de especies fúngicas a partir de unos cuantos gramos de suelo, que la composición y frecuencia de micromicetos en este ambiente es dinámica y cambia considerablemente a través del tiempo, y que los suelos mexicanos encierran una diversidad alta de especies fúngicas que en algunos casos constituyen nuevos registros para el país.

\section{Agradecimientos}

Se agradece el apoyo financiero brindado durante la elaboración de este trabajo al Programa de Becas para la Elaboración de Tesis de Licenciatura en Proyectos de Investigación (PROBETEL) de la Universidad Nacional Autónoma de México, así como a Marcela Del Olmo, Felipe Ruán y Alejandra Cid por su colaboración durante los muestreos.

\section{Literatura citada}

Alef, K. y P. Nannipieri (eds.). 1995. Methods in applied soil microbiology and biochemistry. Academic, London. 576 p.

Barnett, H. L. y B. B. Hunter. 1998. Illustrated genera of imperfect fungi. $4^{\text {th }}$ edtion. APS, St. Paul, Minnesota. 218 p.

Bills, G. F., R. M. Arias, M. Reyes y G. Heredia. 2001. Merimbla humicoloides sp. nov. from conifer forest soil of Veracruz state, Mexico. Mycological Research 105:1273-1279.

Bissett, J. 1984. A revision of the genus Trichoderma. I. Section Longibrachiatum sect. nov. Canadian Journal of Botany 62:924-931.

Bissett, J. 1991. A revision of the genus Trichoderma. III. Section Pachybasium. Canadian Journal of Botany 69:2373-2417.

Bissett, J. 1991a. A revision of the genus Trichoderma. IV. Additional notes on section Longibrachiatum. Canadian Journal of Botany 69:2418-2420.

Booth, C. 1977. Fusarium. Laboratory guide to the identification of the major species.Commonwealth Mycological Institute, Kew, Surrey, England. 58 p.

CEA (Centro de Estadística Agropecuaria). 1999. Anuario estadístico de la producción agrícola de los Estados Unidos Mexicanos. Secretaría de Agricultura, Ganadería, Desarrollo Rural, Pesca y Alimentación, México, D.F. 200 p.

Céspedes, A. E. y J. Castillo. 1982. Algunos Chytridiomycetes y Oomycetes aislados de 10 localidades en cuatro estados de la República Mexicana. Boletín de la Sociedad Mexicana de Micología 17:207-215.

Christensen, M. 1981. Species diversity and dominance in fungal communities. In The fungal community. Its organization and role in the ecosystem, D. T. Wicklow y G. C. Carroll (eds.). Marcel Dekker, New York. p. 201-232. 
Davet, P. y F. Rouxel. 2000. Detection and isolation of soil fungi. Science, Enfield, New Hampshire. 188 p.

Domsch, L. H. y W. Gams. 1972. Fungi in agricultural soils. Halsted, New York. 290 p.

Domsch, K. H., W. Gams y T.H. Anderson. 1993. Compendium of soil fungi. IHW-Verlag, Regensburg, Alemania. 859 p.

Farrow, W. M. 1954. Tropical soil fungi. Mycologia 46:632646.

Heredia, G. 1999. Diversidad y sucesión de los hyphomycetes de la superficie de las hojas en descomposición de tres especies arbóreas dominantes en un bosque mesófilo de montaña en el centro de Veracruz. Tesis doctorado, Facultad de Ciencias, Universidad Nacional Autónoma de México. México, D.F. 499 p.

Heredia, G., M. Reyes, R.M. Arias y G.F. Bills. 2001. Talaromyces ocotl sp. nov. and observations on T. rotundus from conifer forest soils of Veracruz state, Mexico. Mycologia 93:528540.

Ho, M., R. F. Castañeda y F. M. Dugan. 1999. Cladosporium and Cladophialophora in culture: descriptions and an expanded key. Mycotaxon 72:115-157.

INEGI (Instituto Nacional de Estadística, Geografía e Informática) 2001. Síntesis de Información Geográfica del Estado de Tabasco, Aguascalientes, Aguascalientes. 100 p.

Joffe, A. Z. 1963. The mycoflora of a continuously cropped soil in Israel, with special reference to effects of manuring and fertilizing. Mycologia 55:271-282.

Joshi, I. J. y R. K. S. Chauhan. 1982. Investigations into the soil mycoecology of Chambal ravines of India. Plant and Soil 66:329-338.

Klich, M. A. y J. I. Pitt. 1988. A laboratory guide to common Aspergillus species and their teleomorphs. Commonwealth Scientific and Industrial Research Organisation, Australia. $116 \mathrm{p}$.

Mueller-Dombois, D. 1981. Ecological measurements and microbial populations. In The fungal community. Its organization and role in the ecosystem, D. T. Wicklow y G. C. Carroll (eds.). Marcel Dekker, New York. p. 123-184.

Nelson, P. E., T. A. Toussoun y W. F. O. Marasas. 1983. Fusarium species. An illustrated manual for identification. Pennsylvania State University Press, Pennsylvania. 193 p.

Pfenning, L. 1996. Soil and rhizosphere microfungi from Brazilian tropical forest ecosystems. In Biodiversity of tropical microfungi, H. D. Hyde (ed.). Hong Kong University Press. p. 341-365.

Piñón, G. G. 1984. Comunidades fúngicas de los suelos de La Joya del Obispo, Oaxaca. Tesis, Facultad de Ciencias, Universidad Nacional Autónoma de México, México, D.F. $59 \mathrm{p}$.

Pitt, J. I. 2000. A laboratory guide to common Penicillium species. Food Science Australia, North Ryde, Australia. $197 \mathrm{p}$.

Ranzoni, F. V. 1968. Fungi isolated in culture from soils of the Sonora desert. Mycologia 60:356-371.

Raper, K. B. y D. I. Fennell. 1965. The genus Aspergillus. Williams \& Wilkins, Baltimore, Maryland. 686 p.

Reyes, J. y J. Castillo, J. 1981. Micromicetos de la rizósfera del sorgo. Boletín de la Sociedad Mexicana de Micología 15:5-8.

Rodríguez, C. 1984. Comunidades fúngicas de suelos derivados de cenizas volcánicas. Tesis, Facultad de Ciencias, Universidad Nacional Autónoma de México, México, D.F. $45 \mathrm{p}$.

Rosique, E. 2004. Diversidad y abundancia de los hongos microscópicos del suelo de un tintal de Tabasco, México. Tesis, Maestría Facultad de Ciencias, Universidad Nacional Autónoma de México. México, D.F. 116 p.

Samaniego, J.A., M. Ulloa y T. Herrera. 1988. Micobiota del suelo en huertas de nogal atacadas por Phymatotrichum omnivorum en Coahuila, México. Revista Mexicana de Micología 4:43-57.

Shanor, L. 1942. A new Monoblepharella from Mexico. Mycologia 34:241-247.

Velásquez, C. 1989. Aislamiento e identificación de hongos fitopatógenos de raíz de trigo y de suelo en cuatro localidades del Estado de México. Tesis, Facultad de Ciencias, Universidad Nacional Autónoma de México, México, D.F. $53 \mathrm{p}$.

Wicklow, D. T. y W. F. Whittingham. 1974. Soil microfungal changes among the profiles of disturbed conifer- hardwood forests. Ecology 55:3-16.

Wolf, F. T. 1939. A study of some aquatic phycomycetes isolated from mexican soils. Mycologia 31:376-387.

Zambrano, G. y C. Casas- Campillo. 1959. Presencia y contenido de levaduras en suelos tropicales de México. Revista Latinoamericana de Microbiología 2:77-88. 